Rev. Adm. Saúde Vol. 17, № 68, Jul. - Set. 2017

http://dx.doi.org/10.23973/ras.68.55

ARTIGO DE REVISÃO

\title{
Análise dos registros realizados pela enfermagem e o possível impacto na auditoria: uma revisão da literatura nacional
}

Analysis of registries performed by nursing and possible audit impact: a review of national literature

\section{Lúcio Rodrigo Lucca de Camargo ${ }^{1}$, Giovana Rodrigues Pereira ${ }^{2}$}

1. Enfermeiro, pós-graduado em auditoria em saúde. Professor do curso técnico de enfermagem da Faculdade Factum

2. Farmacêutica, especialização em administração hospitalar. Aluna do PPG de pneumologia da Faculdade de Medicina do Hospital de Clínicas/UFRGS

\section{RESUMO}

A auditoria em saúde é uma prática que serve para melhorar a qualidade do serviço prestado, bem como possibilitar que os processos e procedimentos não causem prejuízo ao paciente. Para isto é importante que a enfermagem realize os registros de todas ações, sempre de forma completa e com a identificação do profissional. O objetivo deste trabalho é analisar os registros realizados pela enfermagem encontrados na literatura nacional e descrever possíveis impactos na auditoria. Para isto foi realizado uma revisão da literatura, buscando artigos publicados nas bases de dados LILACS e SCIELO nos últimos 10 anos (2006 a 2016), em português e disponíveis para acesso gratuito. Foram selecionados nove artigos. Todos apontavam inconsistência nos registros, relatando como principais problemas as inconsistências e incoerências nos dados, bem como, a ausência de identificação, horário específico, rasuras e anotações incompletas, que comprometem muito a qualidade da assistência. Outra grande dificuldade encontrada para realização do estudo foi a carência de artigos que tratem sobre o assunto.

Palavras-chave: auditoria de enfermagem, registros de enfermagem, assistência à saúde

\footnotetext{
${ }^{1}$ Enfermeiro pós-graduando em Auditoria em Saúde pelo IBGEN. Email: Irlcamargo@yahoo.com.br

2Professora e Orientadora TCC IBGEN/SEG. Email: professora.giovana.rs@gmail.com
} 


\begin{abstract}
Healthcare auditing is a practice that improves the quality of the service provided, as well as facilitates processes and procedures not to cause harm to the population. To make it possible, it is important that the nursing staff record all their actions, always in a fully way with the identification of the professional. The aim of this study is to analyze the nursing records described in the national literature and describe possible impacts on the auditing. A review of the literature was carried out, searching for articles published in the LILACS and SCIELO databases in the last 10 years (2006 to 2016), in Portuguese language and for free access. Nine articles were chosen, all of which pointed out inconsistencies in the records, describing as the main problems the data incoherencies, as well as the lack of identification, accurate time, erasures an incomplete information, that undermine the quality of healthcare. Another main difficulty found to make this study possible was the lack of articles regarding this subject.
\end{abstract}

Keywords: nursing audit, nursing records, health care

\title{
INTRODUÇÃO
}

Quando pensamos em análise de contas, e em especial as que dizem respeito à assistência em saúde, somos remetidos a auditoria, que surge primeiramente na profissão da contabilidade de forma gerencial e trazendo subsídios para realizar o levantamento de despesas e receitas buscando principalmente identificar inconformidades e posteriormente planejando as adequações necessárias.

A palavra auditoria tem origem do Latim Audire, que significa ouvir, mas o que melhor representa o seu significado encontra-se extraído da língua inglesa, Audit, examinar, corrigir, certificar. É uma especialização da contabilidade que pode ser utilizada por outras profissões, com a finalidade de avaliar os serviços prestados, bem como o controle do patrimônio ${ }^{1}$.

Para os autores que trabalham com a contabilidade, a auditoria consiste em uma técnica sistemática de exame dos registros, que visa demonstrar as situações patrimoniais trazendo críticas, opiniões e reflexões acerca dos resultados apresentados ${ }^{2}$.

Muitas empresas de vários segmentos têm buscado na auditoria uma forma de garantir a qualidade dos serviços e concomitantemente diminuir custos para se tornarem cada vez mais competitivos dentro do mercado, sem falar que em um país cada vez mais corrupto, a auditoria pode trazer uma imagem mais positiva e idônea daquele serviço para sociedade.

Segundo Camelo, Pinheiro, Campos, Oliveira" ${ }^{3}$ "a fim de garantir a qualidade dos serviços prestados aos clientes, nos dias atuais, grandes empresas têm-se 
preocupado em utilizar a auditoria, de forma contínua em suas organizações, visto que os clientes estão cada vez mais convictos de seus direitos".

De acordo com Crepaldi ${ }^{4}$, "a auditoria constitui um conjunto de procedimentos técnicos que tem por objetivo a emissão de parecer sobre sua adequação, consoante os Princípios Fundamentais de Contabilidade e pertinente a legislação específica". Observa-se, portanto a importância de se utilizar com eficácia na busca de possíveis falhas nas contas auditadas diminuindo com isto os custos e principalmente as perdas financeiras nos serviços de várias profissões.

Para Chiavenato 5 ,"a auditoria é um sistema de revisão e controle, para informar a administração sobre a eficiência e eficácia dos programas em desenvolvimento. Sua função não é somente indicar as falhas e os problemas, mas também, apontar sugestões e soluções, assumindo, portanto, um caráter eminentemente educacional".

A auditoria desponta então como uma importante ferramenta para mensurar a qualidade (auditoria de cuidados) e custos (auditoria de custos) das instituições de saúde, sendo que Rodrigues, Perroca, Jericó ${ }^{6}$ conceituam o processo de auditoria como uma avaliação sistemática e formal de uma atividade realizada por pessoas não envolvidas diretamente em sua execução, fiscalizando, controlando, avaliando, regulando e otimizando a utilização de recursos, físicos e humanos, a fim de determinar se a atividade está de acordo com os objetivos propostos.

"Em saúde, a auditoria tem ampliado seu campo de atuação para a análise da assistência prestada, tendo em vista a qualidade e seus envolvidos, que são pacientes, hospital e operadora de saúde, conferindo os procedimentos executados com os valores cobrados, para garantir um pagamento justo"1.

O enfermeiro é preparado desde a graduação a ter uma visão holística tanto de cunho gerencial quanto assistencial, com conhecimento técnico científico, essenciais para fundamentar as ações e condutas realizadas, com isto consegue identificar as inconformidades precocemente, bem como elaborar um plano de ação para melhorar a qualidade do serviço e diminuir os custos do mesmo, o que é uma vantagem quando falamos em auditoria de contas hospitalares.

"Dentro da área da saúde, as enfermeiras são profissionais que possuem uma visão administrativa, dado que são responsáveis pelo gerenciamento da assistência prestada aos usuários. Além disso, detêm conhecimento sobre o uso de medicamentos e materiais, que são de grande valor para o foco que predomina na auditoria privada em saúde, ou seja, o controle de custos" ${ }^{2}$.

Já os autores Faraco, Albuquerque ${ }^{7}$ colocam que o enfermeiro auditor desenvolve habilidades inerentes ao processo, como identificar novos e antigos problemas e apontar as alternativas para realização das devidas correções, além de definir métodos preventivos para diminuir as chances de novas ocorrências. Um benefício não somente para o paciente, mas também para as instituições de saúde e planos de saúde.

Em outro estudo publicado por Siqueira ${ }^{8}$, a autora coloca que o enfermeiro auditor é de suma importância quando o propósito é diminuir custos, evitar desperdícios e 
melhorar a qualidade da assistência prestada, trazendo inúmeros benefícios para a instituição e para o paciente.

A auditoria em enfermagem surgiu como uma ferramenta para identificar falhas nos processos e propor formas de correção, portanto, é definida como um modo de avaliação sistêmica da qualidade de assistência de enfermagem prestada ao paciente, podendo ser pela análise do prontuário, denominada como Auditoria Retrospectiva, pelo acompanhamento ao enfermo "in loco" definido como Auditoria Concorrente, ou pela avaliação prévia dos procedimentos a serem executados, definida como Auditoria Prospectiva?

Porém para Possari ${ }^{10}$ a auditoria em enfermagem pode ser retrospectiva, a qual é realizada após a alta do paciente, utilizando assim o prontuário para avaliação, buscando sempre elementos que possam evidenciar os fatos. Já a operacional ou concorrente, é feita enquanto o paciente está hospitalizado ou em atendimento ambulatorial, onde existe uma revisão dos cuidados prestados, podendo identificar falhas e apontar correções no momento de sua identificação.

Conforme podemos identificar, em qualquer que seja o tipo de auditoria a ser realizada, e para termos êxito, devemos contar com registros e anotações de enfermagem completas e corretas, para se ter embasamento e fundamentação na tomada de decisão durante o processo de auditoria, fazendo parte do prontuário do paciente.

O prontuário médico é uma importante ferramenta legal na avaliação da qualidade da assistência prestada ao cliente, pois se trata de um conjunto de documentos padronizados e organizados, destinados ao registro dos cuidados prestados pelos profissionais de saúde envolvidos no cuidado. Portanto, anotações ou registros que possam gerar dúvidas nos procedimentos realizados, ou a falta de anotações de enfermagem, podem acarretar em glosas de contas hospitalares, gerando com isto perda financeira ${ }^{11}$.

Buscando a forma mais adequada de se realizar o registro, bem como orientar e normatizar os profissionais de enfermagem, o Conselho Federal de Enfermagem, publicou a Resolução no 514/2016 ${ }^{12}$, que "Aprova o Guia de Recomendações para os registros de enfermagem no prontuário do paciente, com a finalidade de nortear os profissionais de Enfermagem". Um manual que discorre sobre o que e como descrever as ações de enfermagem realizadas durante o exercício profissional, uma forma também de respaldar os trabalhadores.

As anotações de forma correta, que trazem todas as informações necessárias ao cuidado prestado, estão muito aquém do que se preconizam, alguns estudos demonstram as carências de informações, que podem acarretar perdas significantes, tanto de foro financeira, quanto de qualidade da assistência.

Segundo Stez, D'Innocenzo ${ }^{13}$ a realização dos registros de forma completa e correta, respalda ética e legalmente o profissional responsável pelo cuidado prestado, assim como o próprio paciente, porém, quando esse registro é escasso e inadequado, compromete toda cadeia de cuidado, o paciente, a instituição e a equipe de enfermagem. 
"A qualidade dos registros sobre o atendimento prestado aos pacientes em situações de emergência é uma preocupação crescente nas instituições, considerando que informações insuficientes afetam e interpõem-se à qualidade do cuidado realizado, principalmente quando relacionado aos dados de identificação que, quando incompletos, podem comprometer a segurança do paciente" 14 .

Ferreira, Braga, Valente, Souza, Alves ${ }^{15}$, colocam que as anotações de enfermagem devem ser encaradas pelos profissionais como algo extremamente importante, bem como as implicações decorrentes do preenchimento incorreto destes, e não como um simples cumprimento de norma burocrática.

O preenchimento incorreto das ações realizadas e registradas no prontuário leva a desassistência por parte da equipe que atua diretamente na prestação do cuidado aos pacientes, e podem acarretar em glosas das contas hospitalares, com isto, perda significativa no repasse de verbas.

Portanto, a partir desta preocupação com a forma correta e completa de realizar os registros de enfermagem, a fim de evitar ao máximo as glosas de contas hospitalares e consequentemente as perdas financeiras, é que surge a questão norteadora do estudo: Quais são as principais carências encontradas nos registros de enfermagem, apontadas na literatura nacional?

O objetivo do estudo é analisar os registros realizados pela enfermagem encontrados na literatura nacional e descrever possíveis impactos na auditoria.

\section{METODOLOGIA}

A característica deste estudo é uma pesquisa qualitativa de revisão da literatura que, segundo $\mathrm{Gil}^{16}$, é desenvolvida com material já elaborado, composto principalmente de livros e artigos científicos. A pesquisa de revisão da literatura permite ao pesquisador a cobertura de uma série de fenômenos muito mais ampla do que aquela que poderia pesquisar diretamente.

Foram estabelecidas como fonte de dados para análise neste estudo, duas bases de dados que, atualmente, publicam diversos estudos científicos na área da saúde no Brasil e na América Latina, foram elas a LILACS (Literatura Latino-Americana e do Caribe em Ciências da Saúde) e SCIELO (Scientific Electronic Library Online).

A LILACS é um abrangente índice da literatura científica e técnica da América Latina e Caribe, criada há 27 anos na intenção de contribuir para o aumento da visibilidade, acesso e qualidade da informação em saúde da região.

SCIELO é um produto de cooperação entre a FAPESP (Fundação de Amparo à Pesquisa do Estado de São Paulo) e BIREME (Centro Latino-Americano e do Caribe de Informação em Ciências da Saúde). É um modelo para a publicação eletrônica cooperativa de periódicos científicos na Internet, desenvolvido para responder as necessidades da comunicação científica nos países em desenvolvimento e particularmente na América Latina e Caribe. 
Em relação à seleção dos artigos, foram incluídos todos os estudos encontrados referentes ao tema proposto, publicados nas bases de dados já citadas nos últimos 10 anos, ou seja, no período de 2006 a 2016, sob os seguintes descritores: auditoria de enfermagem, registros de enfermagem e assistência à saúde. Após realização do cruzamento dos descritores nas bases de dados utilizadas, encontramos um total de dez artigos na LILACS e três artigos na SCIELO, sendo que destes, um não estava disponível para acesso gratuito e três não possuíam informações relevantes ao tema proposto no título ou na leitura do resumo. Desta forma, foram selecionados nove estudos para composição desta revisão.

Para cada texto selecionado foi preenchida uma ficha contendo os seguintes itens para análise: (1) objetivo; (2) autores; (3) instituição; (4) descritores; (5) local de estudo; (6) ano de estudo; (7) principais erros encontrados dos registros apontados nos estudos.

A análise dos dados qualitativos foi realizada através de categorização temática. Minayo ${ }^{17}$ afirma que fazer uma análise de dados qualitativos consiste na descoberta do sentido da informação que compõem uma comunicação cuja presença ou frequência signifiquem alguma coisa para o objetivo analítico visado.

Partindo da operacionalização proposta por Minayo ${ }^{17}$, em uma primeira etapa se procedeu à organização do material, procurando identificar tendências e ideias relevantes. Partindo da leitura e releitura, com o propósito de encontrar aspectos que se repetiram ou se destacaram, para então proceder ao preenchimento das fichas de leitura.

A seguir, através de nova exploração do material foram criadas categorias da análise, estas categorias se referem a palavras ou frases que dão uma noção de sentido as informações contidas nos dados empíricos.

Esta pesquisa respeitou a Lei do Direito Autoral n. 9.610, de 19 de fevereiro de 1998, que exige atentar para o referenciamento dos autores das obras utilizadas para 0 estudo ${ }^{18}$.

\section{RESULTADOS E DISCUSSÃO}

Primeiramente foi identificada principalmente uma escassez muito grande de estudos sobre o assunto, o que acarreta uma deficiência de informações sobre a qualidade dos registros em enfermagem, conduta necessária para prestação de cuidado e identificação da evolução do paciente, bem como anotações incompletas podem gerar glosas de contas e perdas financeiras para as instituições. Foram identificados quatro estudos da região sul, quatro da região sudeste e um da região nordeste, quanto ao ano de publicação, quatro foram publicados em 2013, dois em 2008, um em 2009, um em 2012 e um em 2016. Destes, dois foram de revisão integrativa e os demais, sete foram realizados em instituições de saúde. Com isto, podemos identificar que existem duas regiões que se preocupam mais com a qualidade dos registros, e que em 2013, foi a maior produção sobre o tema, o que pode ter sido impulsionado pela publicação da Resolução Cofen $n^{\circ}$ 429/2012 ${ }^{19}$, "Dispõe sobre o registro das ações profissionais no prontuário do paciente, e em 
outros documentos próprios da enfermagem, independente do meio de suporte tradicional ou eletrônico".

Dos nove estudos que compõem este trabalho, oito possuíam auditoria de enfermagem como descritor, e sete possuíam registros de enfermagem na lista de descritores, isto porque para responder a questão deste estudo é necessário que os artigos tratem sobre registros de enfermagem, bem como auditoria de enfermagem.

Quanto à qualidade dos registros apontados nos estudos, todos os nove artigos identificaram ou apontaram falhas nas anotações de enfermagem, sendo elas informações insuficientes, inexistentes ou ineficazes para prática assistencial.

"Frente aos resultados da pesquisa, observamos ainda a fragilidade dos registros executados pela equipe de enfermagem no prontuário, sendo que em muitos casos os dados são insuficientes ou inexistentes"'.

Em outro estudo realizado por Valença, Azevedo, Oliveira, Medeiros, Malveira, Germano ${ }^{20}$, os autores apontam que a auditoria em saúde surgiu a partir de uma necessidade de avaliação da qualidade da assistência, o que somente será possível através de registros fidedignos da prestação do cuidado, que, para tanto, deve apresentar informações completas, legíveis e fidedignas.

Frente às custas hospitalares e glosas de contas, que podem gerar um déficit financeiro importante para as instituições de saúde, os autores Godoi, Machado, Lins, Cruz, Batista, Rosa ${ }^{21}$ procuraram identificar os principais indicadores responsáveis pelo maior número de glosas relacionadas as anotações de enfermagem, e concluíram que existe uma inconformidade entre o conteúdo das prescrições médicas com os procedimentos executados durante o período hospitalar, bem como apresentaram dados não concisos e incompletos, destacando principalmente falta de orientação ao paciente e familiares quanto a terapêutica e alta hospitalar. Quanto aos indicadores de glosas, que geram perda financeira, as maiores divergências foram em relação à identificação da checagem, identificação da evolução e identificação do executor da atividade, sendo a categoria de enfermagem a que apresentou maior índice de divergência na instituição hospitalar.

A auditoria de enfermagem é utilizada pelas instituições de saúde com a finalidade de avaliar a qualidade da assistência e acompanhar os custos decorrentes da prestação dos serviços. Observa-se uma deficiência na atuação de enfermagem diante das anotações que constituem na única forma de averiguar o cuidado dessa categoria profissional. [...] Em relação à qualidade dos registros, observou-se que tais anotações são incompletas e não proporcionam a qualidade necessária para oferecer subsídios para análise da auditoria de enfermagem ${ }^{22}$.

Nos artigos analisados foram identificados a falta de letra legível, rasuras, uso de corretor gráfico e anotações por turno como os principais erros encontrados nos registros, que foram também a principal razão da não identificação do horário e as datas das anotações de enfermagem, bem como registros incompletos e incoerentes quanto a prescrição de enfermagem ${ }^{23}$. 


\section{CONCLUSÃO}

Ao buscar estudos científicos que apontassem as deficiências nos registros de enfermagem, encontramos somente nove que contemplavam os critérios previamente estabelecidos, e ao analisar os mesmos, identificamos que todos apontavam carências importantes nos registros executados pelos profissionais de enfermagem, trazendo principalmente inconsistências e incoerências com os dados analisados. Apontaram ainda a ausência de identificação, horário específico, rasuras e anotações incompletas dentre a prevalência de erros.

Os profissionais de enfermagem devem ser orientados através de capacitações periódicas, bem como acompanhamento "in loco" dos registros e anotações de enfermagem, para identificar e sanar os principais erros durante o processo, para que fique clara a importância e a correta forma de descrever no prontuário do paciente as condutas realizadas e a terapêutica utilizada desde a internação até a alta hospitalar, somente desta forma poderemos melhorar a qualidade da assistência e diminuir perdas financeiras para as instituições hospitalares. Os profissionais devem conhecer e se apropriar das legislações vigentes quanto à forma correta e a importância dos registros para respaldo das ações executadas.

A auditoria em enfermagem tem como papel principal identificar carências e propor condutas para melhorar a qualidade de assistência, diminuir as glosas nas contas hospitalares e consequentemente melhorar o faturamento diminuindo as perdas financeiras, mas para isto, precisamos de estudos que possam subsidiar as ações de enfermagem, que devem ser pautadas em evidência cientifica, o que se constatou foi uma carência de estudos, o que dificulta o crescimento do conhecimento profissional.

\section{REFERÊNCIAS}

1. LUZ A, MARTINS AP, DYNEWICZ AM. Características de anotações de enfermagem encontradas em auditoria. Revista Eletrônica de Enfermagem, v.09, n.2, 2007. Disponível em: <http://www.fen.ufg.br/revista/v9/n2/v9n2a05.htm>. Acesso em: 05 de maio de 2017.

2. PINTO KA, MELO MM. A prática da enfermeira em auditoria em saúde. Revista Escola Enfermagem USP, v.44, n.3, 2010. Disponível em: $<$ http://www.scielo.br/pdf/reeusp/v44n3/17.pdf >. Acesso em: 05 de maio de 2017.

3. CAMELO SHH, PINHEIRO A, CAMPOS D, OLIVEIRA TL. Auditoria de enfermagem e a qualidade da assistência à saúde: uma revisão da literatura. Revista Eletrônica de Enfermagem, v.11, n.4, 2009. Disponível em: $<$ http://www.fen.ufg.br/revista/v11/n4/v11n4a28.htm >. Acesso em: 05 de maio de 2017.

4. CREPALDI S A. Auditoria contábil: teoria e prática. 4. ed. São Paulo: Atlas, 2007.

5. CHIAVENATO I. Administração de recursos humanos. 2. Ed. São Paulo: Atlas, 1981. 3.v. 
6. RODRIGUES VA, PERROCA MG, JERICÓ MC. Glosas hospitalares: importância das anotações de enfermagem. Arquivos de ciências da Saúde, v.11, n.4, 2004.

Disponível em:

<http://www.rdconsultoria.com.br/Downloads/Educa\%C3\%A7\%C3\%A30\%20Continu ada/Administra\%C3\%A7\%C3\%A30\%2OHospitalar/Glosas\%20Hospitalares.pdf>. Acesso em: 05 de Maio de 2017.

7. FARACO MM, ALBUQUERQUE GL. Auditoria do método de assistência de enfermagem. Revista Brasileira de Enfermagem, v.57, n.4, 2004. Disponível em: $<$ http://www.scielo.br/scielo.php?pid=S0034-

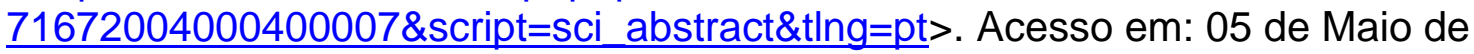
2017.

8. SIQUEIRA PLF. Auditoria em saúde e atribuições do enfermeiro auditor. Caderno saúde e desenvolvimento, v.3, n.2, 2014. Disponível em:

$<$ https://www.uninter.com/revistasaude/index.php/cadernosaudedesenvolvimento/arti cle/download/303/234>. Acesso em: 05 de Maio de 2017.

9. BLANK CY, SANCHES EM, LEOPARDI MT. A prática do enfermeiro auditor hospitalar na região do vale do Itajaí. Revista Eletrônica de Enfermagem, v.15, n.1. Disponível em: http://dx.doi.org/10.5216/ree.v15i1.15082>. Acesso em: 05 de Maio de 2017.

10. POSSARI I. Prontuário do paciente e os registros de enfermagem. 1. ed. São Paulo: látria, 2005.

11. Conselho Federal de Medicina. Código de ética dos profissionais de medicina [online]. Resolução 1331/89. Diário Oficial da União 25 de setembro de 1989; Seção $1 ;$ p.17145.

12. Conselho Federal de Enfermagem. Resolução ํㅡ 514/2016. Aprova o Guia de Recomendações para os registros de enfermagem no prontuário do paciente, com a finalidade de nortear os profissionais de Enfermagem. Diário Oficial da União 07 de junho de 2016; Seção 1; p.84

13. STEZ VG, D'INNOCENZO M. Avaliação da qualidade dos registros de enfermagem no prontuário por meio da auditoria. Acta Paulista de Enfermagem, v.22, n.3, 2009. Disponível em: <http://www.scielo.br/pdf/ape/v22n3/a12v22n3.pdf>. Acesso em: 08 de Maio de 2017.

14. SILVA JA, GROSSI ACM, HADDAD MCL, MARCON SS. Avaliação da qualidade das anotações de enfermagem em unidade semi-intensiva. Escola Ana Nery, v.16, n.3, 2012. Disponível em :

$<$ http://www.scielo.br/scielo.php?script=sci arttext\&pid=S141481452012000300021>. Acesso em: 08 de Maio de 2017.

15. FERREIRA TS, BRAGA ALS, VALENTE GSC, SOUZA DF, ALVES EMC. Auditoria de enfermagem: o impacto das anotações de enfermagem no contexto das glosas hospitalares. AÑO 9, v.9, n.1, Chia/Colômbia, 2009. Disponível em: <http://www.uff.br/anaissegerenf/premio/AUDITORIA\%20EM\%20ENFERMAGEM\%2 0O\%20IMPACTO\%20DAS\%20ANOTA\%C7\%D5ES\%20DE\%20ENFERMAGEM\%20 
NO\%20CONTEXTO\%20DAS\%20GLOSAS\%20HOSPITALARES.pdf>. Acesso em: 08 de Maio de 2017.

16. GIL AC. Como elaborar projetos de pesquisa. 4. Ed. São Paulo: Atlas, 2007.

17. MINAYO MC. O desafio do conhecimento: pesquisa qualitativa em saúde. Rio de Janeiro: Abrasco, 2007.

18. BRASIL, Lei no 9.610, de 19 de fevereiro de 1998. Presidência da República Casa Civil, Subchefe para Assuntos Jurídicos. Poder Judiciário, Brasília, DF, 1998.

19. Conselho Federal de Enfermagem. Resolução nํ4 429/2012. Dispõe sobre o registro das ações profissionais no prontuário do paciente, e em outros documentos próprios da enfermagem, independente do meio de suporte - tradicional ou eletrônico. Diário Oficial da União 08 de junho de 2012; Seção 1; p.288.

20. VALENÇA CN, AZEVEDO LMN, OLIVEIRA AG, MEDEIROS SSA, MALVEIRA FAZ, GERMANO RM. A produção científica sobre auditoria de enfermagem e qualidade dos registros. Revista de Pesquisa Cuidado é Fundamental Online, v.5, n.5, 2013. Disponível em:

<http://www.seer.unirio.br/index.php/cuidadofundamental/article/view/1617/pdf 996>. Acesso em: 10 de Maio de 2017.

21. GODOI AP, MACHADO CS, LINS MA, CRUZ MG, BATISTA VM, ROSA BA. Auditoria de custo: análise comparativa das evidências de glosas em prontuário hospitalar. Revista do Instituto de Ciências da Saúde, v.26, n.4, 2008. Disponível em:

$<$ https://www.unip.br/comunicacao/publicacoes/ics/edicoes/2008/04 out dez/V26 N 4 p403-408.pdf >. Acesso em 10 de Maio de 2017.

22. OLIVEIRA DR, JACINTO SM, SIQUEIRA CL. Auditoria de enfermagem em Centro Cirúrgico. Revista de Administração em Saúde, v.15, n.61, 2013. Disponível em:

$<$ http://www.google.com.br/url?sa=t\&rct=j\&q=\&esrc=s\&source=web\&cd=1\&sqi=2\&ve d=0ahUKEwiR4Jm76v TAhWMKCYKHYt1A4AQFggnMAA\&url=http\%3A\%2F\%2Fw ww.cqh.org.br\%2Fportal\%2Fpag\%2Fanexos\%2Fbaixar.php\%3Fp ndoc\%3D $1021 \% 2$ 6p nanex0\%3D\%2520506\&usg=AFQjCNG9oOqPb -bJeNLX76TgeGso9olRA>. Acesso em: 13 de Maio de 2017.

23. CLAUDINO HG, GOUVEIA EML, SANTOS SR, LOPES MEL. Auditoria em registros de enfermagem: revisão integrativa da literatura. Revista de Enfermagem UERJ, v.21, n.3, 2013. Disponível em:

<http://www.facenf.uerj.br/v21n3/v21n3a20.pdf>. Acesso em: 13 de Maio de 2017.

Recebido: 17 setembro 2017. Publicado: 16 outubro 2017 
Correspondência: Lúcio Rodrigo Lucca de Camargo; Rua Dr. Dário de Bitencourt, 300 - Apto. 403; CEP 91360-390 - Porto Alegre - RS. E-mail:

Irlcamargo@yahoo.com.br

Conflito de Interesses: os autores declararam não haver conflito de interesses.

(C) This is an Open Access article distributed under the terms of the Creative Commons Attribution License, which permits unrestricted use, distribution, and reproduction in any medium, provided the original work is properly cited 